\title{
Thermosensitive supramolecular and colloidal hydrogels via self-assembly modulated by hydrophobized cellulose nanocrystals
}

\author{
Rinat Nigmatullin • Valeria Gabrielli • Juan C. Muñoz-García • \\ Anna E. Lewandowska • Robert Harniman • Yaroslav Z. Khimyak • \\ Jesús Angulo • Stephen J. Eichhorn
}

Received: 25 September 2018/Accepted: 20 December 2018/Published online: 8 January 2019

(C) The Author(s) 2019

\begin{abstract}
Utilization of reversible non-covalent interactions is a versatile design strategy for the development of stimuli responsive soft materials. In this study, hydrophobic interactions were harnessed to assemble water-soluble macromolecules and nanoparticles into a transient hybrid network forming thermosensitive hydrogels with tunable rheological properties. Hybrid hydrogels were built of biopolymer derived components: cellulose nanocrystals (CNCs), nanoparticles of high aspect ratio, and hydroxypropyl methylcellulose (HPMC). To enable polymer/CNC assembly via hydrophobic interactions, the surface of highly hydrophilic CNCs was modified by binding octyl moieties (octyl-CNCs). The amphiphilicity of
\end{abstract}

Electronic supplementary material The online version of this article (https://doi.org/10.1007/s10570-018-02225-8) contains supplementary material, which is available to authorized users.

R. Nigmatullin · A. E. Lewandowska .

S. J. Eichhorn $(\bowtie)$

Bristol Composites Institute (ACCIS), University of

Bristol, Bristol BS8 1TR, UK

e-mail: s.j.eichhorn@bristol.ac.uk

V. Gabrielli · J. C. Muñoz-García ·

Y. Z. Khimyak · J. Angulo

School of Pharmacy, University of East Anglia, Norwich

Research Park, Norwich NR4 7TJ, UK

R. Harniman

School of Chemistry, University of Bristol,

Bristol BS8 1TS, UK
octyl-CNCs was confirmed by surface tension measurements. The molecular and particulate amphiphiles assemble into hybrid networks, which result in stiffer and stronger hydrogels compared to HPMC hydrogels and hydrogels reinforced with hydrophilic CNCs. Hybrid hydrogels retain the ability of HPMC hydrogels to flow under applied shear stress. However, significantly higher viscosity was achieved for HPMC/ octyl-CNCs compared with HPMC/CNCs hydrogels. The inherent thermal response of rheological properties of HPMC hydrogels was further amplified in combination with octyl-CNCs due to temperatureinduced polymer/nanoparticle association via hydrophobic interactions. Saturation transfer difference NMR spectroscopy demonstrated the growth of network-bound water with an increase in temperature, which correlates with the increase of stiffness and viscosity of hydrogels upon heating. Rheological properties of these hybrid hydrogels are defined by the content of the soluble polymer and the CNCs, and it is shown that they can be finely adjusted for a required application. 


\section{Graphical abstract}

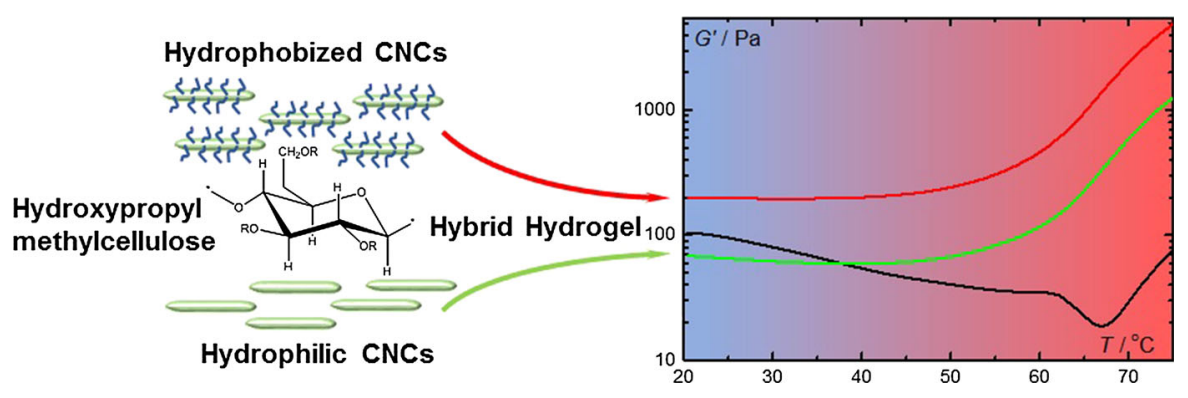

Keywords Cellulose nanocrystals $\cdot$ Hydroxypropyl methylcellulose $\cdot$ Thermoresponsive $\cdot$ Hydrogel

\section{Introduction}

Supramolecular assembly is growing into an established paradigm for the preparation of soft materials. The structural elements in supramolecular networks are held together due to physical cross-links driven by reversible non-covalent interactions such as hydrogen bonding, ionic or hydrophobic interactions, transition metal coordination, $\pi-\pi$ stacking (Appel et al. 2012; Du et al. 2015; Sahoo et al. 2018; Voorhaar and Hoogenboom 2016). The reversible and transient nature of such crosslinking enables the fabrication of environmentally responsive, self-healing, moldable soft materials. These attributes are highly desirable in various applications and actively explored in biomedicine, e.g. injectable hydrogels for tissue regeneration and drug delivery (Sahoo et al. 2018), food and cosmetic industries where encapsulation of bioactive ingredients is required (Zakharova et al. 2016) or for structuring and rheological modification (Zheng and Loh 2016), and the oil industry (Cao et al. 2015), etc.

Supramolecular networks can be built of low molecular weight substances ( $\mathrm{Du}$ et al. 2015) or polymers (Voorhaar and Hoogenboom 2016). However, low molecular weight gelators tend to be complex molecules requiring challenging and costly synthesis. Moreover, the mechanical properties of (hydro)gels formed by small molecules are usually inferior compared to polymer-based counterparts. Polymer-based systems can utilize relatively simple chemical structures, with functional units randomly distributed along the macromolecule. They can also use more sophisticated macromolecular structures such as block co-polymers, polymers functionalized with complex motifs often inspired by biospecific interactions (Appel et al. 2012).

Hydrophobic interactions have been previously used for the improvement of strength and toughness of polymer hydrogels (Mihajlovic et al. 2017; Tuncaboylu et al. 2011) or as associative rheological modifiers in aqueous systems (Chassenieux et al. 2011; Lundberg et al. 1991). This latter approach is based on using copolymers with associative domains as a single hydrophobic block (in ABA-type block copolymers), two end hydrophobic blocks (BAB-type block copolymer) or many randomly distributed hydrophobic groups (Chassenieux et al. 2011). Such amphiphilic copolymers assemble into a variety of morphologies via reversible hydrophobic interactions. Moreover, molecular association involving amphiphilic polymers is sensitive to temperature. This temperature dependence is due to entropically driven release of ordered/bound water at elevated temperatures when hydrophobic domains assemble into the aggregates. Therefore, hydrogels based on amphiphilic polymers provide a platform for the fabrication of reversible self-healing, thermoresponsive hydrogels where their rheology can be tuned from injectable formulations to tough and strong hydrogels.

Further alterations in mechanical properties of hydrogels and diversification of their functional properties can be achieved via the integration of nanoparticles into the hydrogel structure. Nanocomposite hydrogels is a thriving research field, and various nanoparticles have been explored as constituents of hybrid hydrogels. Reflecting the fact that hydrogels are aqueous systems, naturally most nanocomposite hydrogels have been developed with the use of hydrophilic nanoparticles such as clay minerals, 
nanocellulose, silica, graphene oxide (Alam et al. 2018; Haraguchi 2007; Schexnailder and Schmidt 2009). However, Baumann et al. (2010) reported an unexpected increase in stiffness of hydrogels based on a blend of hyaluronic acid and partially hydrophobic methylcellulose (MC) with the addition of hydrophobic nano- and microparticles made of poly(D,L-lacticco-glycolic acid) (PLGA). The authors attributed such an effect to the hydrophobic interactions between MC and PLGA particles. Based on this observation, Appel et al. (2015) proposed the concept of making selfhealing, thermoresponsive supramolecular and colloidal hydrogels via reversible association of soluble macromolecules and particles driven by hydrophobic interactions. To augment hydrophobic interactions, hydroxypropyl methylcellulose (HPMC) was modified using isocyanates with a long alkyl chain (hexyl, adamantyl and dodecyl). The most hydrophobic HPMC modified with dodecyl moieties allowed the formation of much stronger hydrogels in the presence of hydrophobic particles (polystyrene and PEG- $b$ PLA block copolymer). This confirmed that reversible networks can be built via transient hydrophobic interactions between amphiphilic polymers and nanoparticles. Such hydrogels retained shear-thinning properties and quickly restored their network structures after shear deformation.

The current study further explores this concept using nanoparticles of high aspect ratio, cellulose nanocrystals (CNCs). To enable the formation of supramolecular and colloidal networks due to hydrophobic interactions, the surface of the CNCs is modified by binding hydrophobic octyl moieties (octyl-CNCs). HPMC was used as a soluble polymer with amphiphilic properties. Surface activity and thermal response of octyl-CNCs was confirmed by surface tension measurements. These all-cellulose supramolecular and colloidal systems are shear-thinning, temperature sensitive hydrogels of higher stiffness and strength compared to materials made with HPMC alone, and those containing only hydrophilic CNCs.

\section{Materials and methods}

\section{Materials}

Sodium metaperiodate, octylamine, hydroxypropyl methylcellulose (HPMC) with a molecular weight of approximately $86 \mathrm{kDa}$ and viscosity of $-2600-5600$ cP (2 wt $\%$ solution in water) were purchased from Sigma-Aldrich (Loughborough, UK) and used without further purification. Cellulose nanocrystals (CNCs) in sodium form were supplied as an $11.3 \mathrm{wt} \%$ aqueous slurry by Maine University Process Development Centre (USA, Orono, ME). According to the manufacturer, the sulfur content of the CNCs was $0.9 \mathrm{wt} \%$ sulfur per gram of dry CNCs.

Octyl-CNCs were synthesized according to the procedure described elsewhere (Nigmatullin et al. 2018) and in Supplementary Material. In short, the synthesis was made on a scale of $2.5 \mathrm{~g} \mathrm{CNCs}$. CNCs were oxidized by $0.9 \mathrm{~g}$ of $\mathrm{KIO}_{4}$ for $24 \mathrm{~h}$. After, purification oxidized CNCs were reacted with $2.5 \mathrm{~g}$ of octylamine for $3 \mathrm{~h}$ at $45{ }^{\circ} \mathrm{C}$ and for a further $21 \mathrm{~h}$ at room temperature after adding $\mathrm{NaBH}_{3} \mathrm{CN}$. Octyl$\mathrm{CNCs}$ were purified using centrifugation and finally dialyzed against deionized water. The suspension of purified octyl-CNCs was concentrated by allowing water evaporation through the dialysis membrane. Purified octyl-CNCs were kept as never-dried material as a concentrated gel. Chemical transformations of cellulose on the surface of CNCs are presented in Scheme S1 of Supplementary Material. From the analysis of ${ }^{1} \mathrm{H}^{13} \mathrm{C}$ CP-MAS NMR spectra, the degree of surface functionalization was found to be around 4\% (Electronic Supplementary Material, Figure S1, Table S1). AFM images of the topology of CNCs and octyl-CNCs (Electronic Supplementary Material, Figure S2) confirmed a rod-shaped morphology for both parent CNCs and octyl-CNCs. CNC modification did not affect the size of the nanoparticles. For both types of CNCs, their length varied between 50 and $160 \mathrm{~nm}$, while their widths were found to be around $4 \mathrm{~nm}$.

Preparation of hydrogels

$4 \mathrm{wt} \%$ solution of HPMC was used a stock solution for the preparation of hydrogels. The required amount of powdered HPMC was suspended in deionized water and a mixture kept in a media bottle was left under constant rotation using a roller mixer at $60 \mathrm{rpm}$ for $24 \mathrm{~h}$ at room temperature. The clear colorless solution was stored at $6{ }^{\circ} \mathrm{C}$. Both $\mathrm{CNCs}$ and octyl-CNCs were used as never-dried materials in gels with concentration $11.3 \mathrm{wt} \%$ and $5 \mathrm{wt} \%$ respectively. For hydrogel preparation the required amounts of HPMC stock solution, CNC or octyl-CNC gels and deionized water 
were thoroughly mixed manually using a spatula. Freshly prepared hydrogels were used for rheological and SEM studies.

\section{Scanning Electron Cryomicroscopy (Cryo-SEM)}

For imaging hydrogels, a small volume of a sample was placed in the head of a cryo-rivet. A second rivet was balanced on the top of the head and the assembly was frozen in liquid nitrogen slush. The frozen assembly was then transferred under vacuum into a Gatan Alto cryo-SEM transfer system mounted on a FEI Quanta 200 FEG SEM. Here the top rivet was removed and exposed a fractured surface. The frozen sample of a hydrogel underwent sublimation and sputter coating with $\mathrm{Au} / \mathrm{Pd}$. The sample was then transferred to the cooled SEM stage for observation.

\section{Atomic Force Microscopy (AFM)}

AFM has been used for characterization of unmodified and modified CNCs. Samples were drop-cast onto freshly cleaved mica substrates using a diluted suspension with a CNC concentration of $0.02 \mathrm{mg} /$ $\mathrm{mL}$. The suspension was allowed to dry before Scanning Probe Microscopy (SPM) investigation in an ambient environment. SPM measurements were conducted using a Multi-Mode VIII microscope with Nanoscope V controller operating under non-resonant PeakForce feedback control (Bruker, CA, USA). SCANASYST-FLUID + cantilevers were employed with nominal spring constants of $0.7 \mathrm{~N} / \mathrm{m}$ (Bruker, CA, USA).

\section{Surface tension measurements}

An advanced surface tensiometer from Krüss (Germany, model Krüss K100) was used for the measurements of surface tension. For the determination of the surface tension of CMC, a standard measuring probe (PL01) for the Wilhelmy plate method was used. Measurements at different $\mathrm{CNC}$ concentrations were performed by automatic serial dilutions using two micro dispensers (DS0810). The initial concentration of the octyl-CNC suspension was $7000 \mathrm{mg} / \mathrm{L} .200$ measurements were conducted, and the final concentration of octyl-CNCs was $10 \mathrm{mg} / \mathrm{L}$. The CMC value was obtained using semi-log plot of surface tension against concentration. The influence of temperature on surface tension was studied in measurements using a rod-shaped probe (PL03) for the Wilhelmy method and a measuring vessel with capacity of $5 \mathrm{~mL}$. HPMC solutions or octyl-CNC suspensions were heated to $80{ }^{\circ} \mathrm{C}$. An aliquot of the hot solution was transferred into the measuring vessel and continuous measurements of surface tension were conducted for $20 \mathrm{~min}$ while the solution cooled down naturally. The instrument acquired surface tension values together with the corresponding temperature which was measured by an integrated temperature probe.

\section{Raman spectroscopy}

Raman spectra were recorded using a confocal Raman microscope (Alpha300, WITec GmbH), equipped with a thermoelectrically cooled CCD detector (down to $-61{ }^{\circ} \mathrm{C}$ ). A $532 \mathrm{~nm}$ wavelength laser was used for excitation, and a $50 \times$ objective lens was used for backscattered light collection with a lateral resolution of $388 \mathrm{~nm}$. The spectrometer grating was $600 \mathrm{~g} \mathrm{~mm}^{-1}, \mathrm{BLZ}=500 \mathrm{~nm}$. Raman spectra were recorded using an exposure time of $10 \mathrm{~s}$ and 25 accumulations. The sample film was supported on microscope slide. The analysis of Raman spectra included deconvolution based on Lorentz function to estimate the number and position of Raman bands.

Rheological measurements

A Discovery HR-1 rotational rheometer (TA Instrument) equipped with a Peltier plate as a temperature controller was used for studying the rheology of the gels. A 'cone and plate' geometry used for the measurements consisted of a stainless-steel cone of $40 \mathrm{~mm}$ diameter and angle $4^{\circ}$, and the Peltier plate. Frequency sweeps were conducted in strain controlled mode at $1.5 \%$ strain and within an angular frequency range from 0.4 to $100 \mathrm{rad}_{\mathrm{s}}{ }^{-1}$. The thermal response of the hydrogels was studied in an oscillation mode between 20 and $70{ }^{\circ} \mathrm{C}$ with a temperature ramp of $5^{\circ}$ per minute using $1.5 \%$ strain and an angular frequency $6.34 \mathrm{rad} / \mathrm{s}$. Additionally, flow curves were obtained at $20,30,40,50$ and $60{ }^{\circ} \mathrm{C}$ in steady shear within a shear rate range of $0.01-100 \mathrm{~s}^{-1}$. Amplitude sweeps were

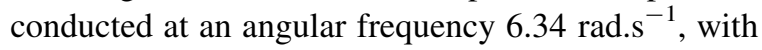
oscillation strain variation between 0.01 and $650 \%$. During the rheological experiments, the measuring 
system was covered with a humidity chamber to minimize water evaporation.

\section{NMR spectroscopy}

HPMC solution and mixtures of HPMC/CNC 50/50 and HPMC/Octyl-CNC 50/50 with total solids content of $1 \mathrm{wt} \%$ were prepared in $\mathrm{D}_{2} \mathrm{O}\left(99.9 \%{ }^{2} \mathrm{H}\right)$ by overnight mixing of the corresponding amounts of the solid materials using a rotary mixer.

Solution state NMR experiments were performed on a Bruker Avance I spectrometer equipped with a $5 \mathrm{~mm}$ probe, operating at a ${ }^{1} \mathrm{H}$ frequency of 499.69 MHz. Around $700 \mu \mathrm{L}$ of sample were pipetted into a $5 \mathrm{~mm}$ NMR tube at room temperature. Variable temperature (VT) experiments were performed at 30 , 40, 55 and $70{ }^{\circ} \mathrm{C}$ with 20 min thermal stabilization before acquisition. Representative spectra obtained at $30{ }^{\circ} \mathrm{C}$ are shown in Figure S3 of Supplementary Material.

To monitor the interactions of water (HDO) with hydrogel components upon heating, saturation transfer difference (STD) NMR experiments (Mayer and Meyer 1999) were performed by selective ${ }^{1} \mathrm{H}$ saturation of the NMR-invisible core of cellulose network (0 ppm) (Calabrese et al. 2019). Cascades of $49 \mathrm{~ms}$ Gaussian-shaped pulses at a field strength of $50 \mathrm{~Hz}$ were employed, with a delay of $4 \mu$ s between successive pulses. The on- and off-resonance frequencies were set to 0 and $50 \mathrm{ppm}$, respectively, and a recycle delay of $6 \mathrm{~s}$ (including the saturation time) was used. The STD spectra were obtained by subtracting the onfrom the off-resonance spectrum ( $\mathrm{I}_{\mathrm{sat}}$ and $\mathrm{I}_{0}$, respectively). To determine the STD response $\left(\eta_{S T D}\right)$ of the HDO peak (which is indicative of the fraction of bound HDO), its signal intensity in the difference spectrum $\left(I_{S T D}\right)$ was integrated relative to the signal intensity in the off-resonance spectrum $\left(I_{0}\right)$, and then by using the equation

$\eta_{S T D}=\frac{I_{0}-I_{\text {sat }}}{I_{0}} \times 100=\frac{I_{S T D}}{I_{0}} \times 100$

Different saturation times $\left(\mathrm{t}_{\mathrm{sat}} ; 0.5,0.75,1,1.5,2,3,4\right.$, 5 and $6 \mathrm{~s}$ ) were used to obtain the STD build-up curve $\left(\mathrm{STD}\left(\mathrm{t}_{\mathrm{sat}}\right) v s \mathrm{t}_{\mathrm{sat}}\right.$ (Figure S4 of Supplementary Material) which was then fitted to the monoexponential equation
$\left.\operatorname{STD}\left(t_{s a t}\right)=\operatorname{STD}^{\max }\left(1-e^{\left(-k_{s a t} * t_{s a t}\right.}\right)\right)$

to derive the parameters $\operatorname{STD}^{\max }$ and $\mathrm{k}_{\mathrm{sat}}$, which represent the asymptotic maximum of the build-up curve and the rate constant of the STD build-up, respectively. The initial slope of the STD build-up curve $\left(\mathrm{STD}_{0}\right)$ was calculated as the product of $\mathrm{STD}^{\max }$ and $\mathrm{k}_{\mathrm{sat}}$, as shown in the equation

$S T D_{0}=\left.\frac{\partial S T D\left(t_{\text {sat }}\right)}{\partial t_{\text {sat }}}\right|_{t_{\text {sat }} \rightarrow 0}=S T D^{\max } * k_{\text {sat }}$

The spectra were analyzed using TopSpin 3.5 software.

\section{Results and discussions}

Surface activity of gel components

It is generally recognized that nanocellulose (fibrils, nanocrystals) are amphiphilic nanoparticles due to exposure of less polar groups on some part of the crystals (Kalashnikova et al. 2012; Mazeau 2011). As a result, various forms of nanocellulose are efficient in the formation of Pickering emulsions (Kalashnikova et al. 2012). It appears that nanocellulose of lower surface charge are more amphiphilic since the efficiency in stabilizing emulsions improves for nanocellulose of low charge. In this work, commercially available CNCs were used. These were produced by hydrolysis in concentrated sulfuric acid and, as a result, they are highly charged (about $230 \mathrm{mmol}$ $-\mathrm{SO}_{3}^{-}$per $\mathrm{kg}$ of $\mathrm{CNCs}$ ). Surface tension measurements for CNCs suspensions with concentrations up to $8 \mathrm{~g} / \mathrm{L}$ deviated from the surface tension of pure water within the error of measurement (data not shown). Thus, unmodified CNCs have very low surface activity. In contrast, hydrophobized octyl-CNCs significantly reduced surface tension (Fig. 1) confirming the surface activity of hydrophobized materials through the attachment of octyl groups. The minimal value of surface tension attainable in octyl-CNC suspension was $\sim 51 \mathrm{mN} / \mathrm{m}$. As can be seen from Fig. 1, there is a strong and linear dependence of surface tension on concentration for octyl-CNC suspensions with concentrations up to approximately $2500 \mathrm{mg} / \mathrm{L}$. This is typical for amphiphiles with concentration-dependent segments at low 


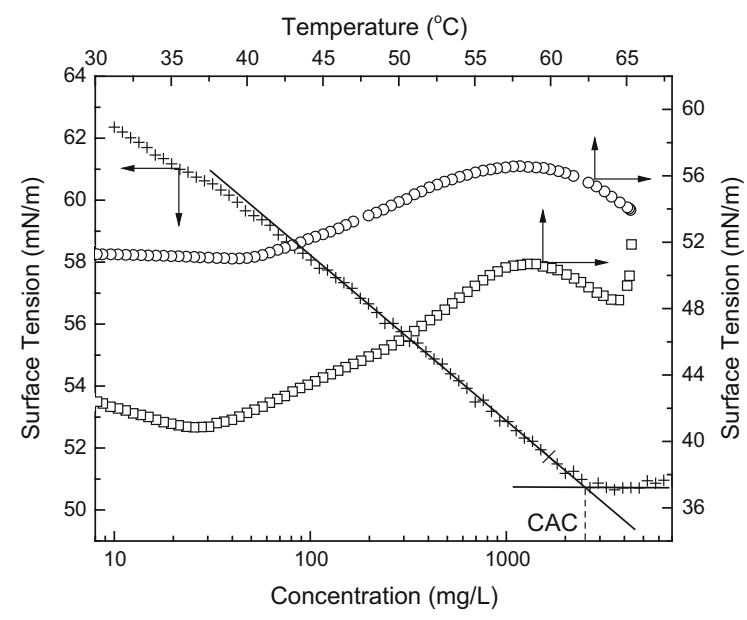

Fig. 1 Dependence of surface tension of octyl-CNC suspensions (cross, circle) and HPMC solution (square) on concentration (cross) and temperature (circle, square). HPMC and octyl$\mathrm{CNC}$ concentration in the temperature dependence study was $500 \mathrm{mg} / \mathrm{L}$. Solid lines are linear fittings of a plateau region and linearly dependent region proceeding the plateau. Critical aggregation concentration (CAC) is determined as an intersection between these fitted lines

concentrations, and a region of constant surface tension at high concentrations. The onset of a steady-state value of surface tension is thought to be attributed to a critical micellar concentration (CMC) or more generally a critical aggregation concentration (CAC) when the morphology of aggregates is not well defined. At this concentration, amphiphiles selfassemble into micellar or aggregated structures in the bulk of the solution (Chakraborty et al. 2011). The CAC for octyl-CNCs was found to be around $2500 \mathrm{mg} / \mathrm{L}$.

Temperature induced association of amphiphiles can be probed by surface tension measurements. To exclude the interference from temperature effects on the size and structure of the dynamic micelles, the dependence of surface tension on temperature of octyl-CNCs was studied using suspensions with a concentration of $500 \mathrm{mg} / \mathrm{L}$, which is well below the CAC of octyl-CNCs. Only a small decrease in surface tension was observed with an increase of temperature to $40{ }^{\circ} \mathrm{C}$ (Fig. 1). After this point, further temperature increases caused a gradual increase in the surface tension. Thus, the state of the octyl-CNCs in the suspension is sensitive to temperature. This indicates that the association of octyl-CNCs in the bulk of the suspension intensifies at temperatures above $40{ }^{\circ} \mathrm{C}$.
This association is thought to cause a decrease in the amount of amphiphilic particles at the air/liquid interface leading to a corresponding increase in surface tension. The decrease in surface tension observed at temperatures above approximately $57{ }^{\circ} \mathrm{C}$ is likely to be due to a combination of two factors: (1) a decrease in the surface tension with temperature characteristic for pure liquids; (2) the dissociation of micelles in the systems with higher thermal energy. To some extent a similar temperature response was observed for the solution of HPMC of the same concentration. HPMC causes a larger decrease in surface tension than octyl-CNCs at the same concentration. Therefore, HPMC is characterized by a higher surface activity. Similar to octyl-CNCs, there was an increase in the surface tension of HPMC between 35 and $57^{\circ} \mathrm{C}$ which is evidence of the molecular association in the HPMC solution within this temperature range. Interestingly, unlike the octyl-CNC suspensions, HPMC solutions underwent a sudden increase in surface tension when the temperature approached $65^{\circ} \mathrm{C}$. At this temperature, extensive association of HPMC macromolecules led to a phase separation, which was noticeable visually as the turbidity of the solution increased dramatically. Thus, from surface tension measurements the temperaturesensitive behavior was observed for both HPMC and octyl-CNCs. It is worth summarizing that the association of these amphiphiles starts at relatively low temperatures (around $40{ }^{\circ} \mathrm{C}$ ) and progresses gradually with an increase in temperature to $60{ }^{\circ} \mathrm{C}$.

\section{Gelation induced by reinforcing with nanoparticles}

Rheology and reversible sol-gel transition for aqueous solutions of HPMC have been studied in detail (Haque et al. 1993; Joshi 2011; Silva et al. 2008). In line with the results of previous studies, the $4 \mathrm{wt} \%$ solution of HPMC used in this study showed a liquid-like behavior in oscillatory measurements at room temperature; in the low frequency zone $G^{\prime \prime}$ was higher than $\mathrm{G}^{\prime}$ (Fig. 2) and there was a sharper growth of $\mathrm{G}^{\prime}$ $\left(G^{\prime} \sim \omega^{1.17}\right)$ compared to $G^{\prime \prime}\left(G^{\prime \prime} \sim \omega^{0.78}\right)$. The curves intersected $\left(G^{\prime}=G^{\prime \prime}\right)$ at an angular frequency of $\sim 40 \mathrm{rad}^{-1}$. Such a response is characteristic for an entangled polymer solution which flows under gravity (Figure S5 of Supplementary Material). However, HPMC solutions with half this concentration (2 

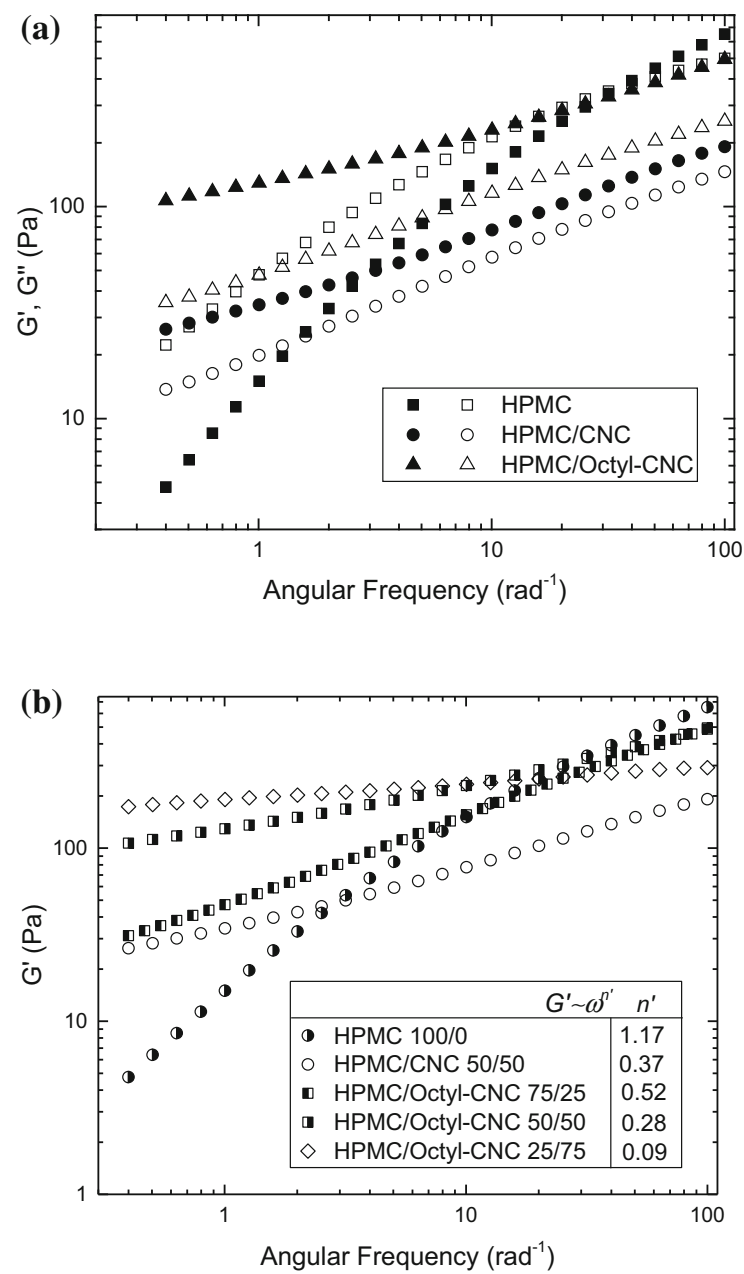

Fig. 2 a Comparison of mechanical spectra of HPMC hydrogel and hybrid hydrogels containing equal amounts of CNCs or octyl-CNC; b the influence of frequency on $\mathrm{G}^{\prime}$ for HPMC hydrogels and hybrid hydrogels of different compositions. Total solids concentration $4 \mathrm{wt} \%$, numbers in compositions represent the mass ratio between HPMC and nanoparticles. Strain amplitude $1.5 \%$

$\mathrm{wt} \%$ ) were transformed to a system with $\mathrm{G}^{\prime}$ dominant compared to $\mathrm{G}^{\prime \prime}$, with the addition of an equal amount of CNCs (total solid content $4 \mathrm{wt} \%$ ). Such a mixture forms invertible hydrogels (Figure S5 of Supplementary Material). With a significant frequency dependence of both $\mathrm{G}^{\prime}\left(\mathrm{G}^{\prime} \sim \omega^{0.37}\right)$ and $\mathrm{G}^{\prime \prime}\left(\mathrm{G}^{\prime \prime} \sim \omega^{0.44}\right)$ HPMC solutions reinforced with CNCs can be defined as a weak gel. Similar effects of CNCs on the rheological properties of methylcellulose (MC), another water soluble thermoresponsive cellulose ether, has been previously reported (Hynninen et al.
2018; McKee et al. 2014). Using isothermal titration calorimetry, it has been shown that the enthalpy of mixing of MC and CNCs is highly negative, confirming an affinity between MC and nanocellulose particles most likely due to hydrogen bonding and van der Waals interactions. This mechanism of formation of a hybrid polymer/nanoparticle network is expected to dominate at low temperatures for the systems with HPMC, since the hydrophilic components of these water-soluble polymers are similar.

As can be seen from Fig. 2, further increase of hydrogel stiffness was observed when CNCs were replaced by hydrophobized octyl-CNCs. In hydrogels with octyl-CNCs instead of CNCs, both $\mathrm{G}^{\prime}$ and $\mathrm{G}^{\prime \prime}$ were less frequency dependent with $\mathrm{G}^{\prime} \sim \omega^{0.28}$ and $\mathrm{G}^{\prime \prime} \sim \omega^{0.37}$. Additionally, differences between $\mathrm{G}^{\prime}$ and $\mathrm{G}^{\prime \prime}$ increases in hydrogels with octyl-CNCs compared with hydrogels reinforced with CNCs. For example, at an angular frequency of $1 \mathrm{~Hz}, \mathrm{G}^{\prime}$ was two times higher than $\mathrm{G}^{\prime \prime}(\tan \delta \approx 0.5)$ in hydrogels with octyl-CNCs but $1.4(\tan \delta \approx 0.7)$ times larger in the hydrogel with CNCs. These observations indicate that the hybrid network formed by HPMC and octyl-CNCs is stronger which could be a result of an increased number of transient physical cross-links or/and an increased strength of interaction between the bridging soluble polymer and nanoparticles. This strongly suggests that association between hydrophobic domains of octylCNCs and HPMC modulates the stiffening of the hydrogels reinforced with particles according to the approach proposed by Appel et al. (2015).

In an attempt to elucidate differences in interactions between HPMC and hydrophilic or hydrophobic CNC, films of HPMC/CNC and HPMC/octyl-CNC composites were studied in comparison with HPMC and CNC or octyl-CNC films using Raman spectroscopy. Addition of either CNCs or octyl-CNCs to HPMC had little effect on the positions of the main HPMC bands (Figure S6a of Supplementary Material). However, the HPMC band, located at $\sim 947 \mathrm{~cm}^{-1}$ which is ascribed to the $\mathrm{C}-\mathrm{O}-\mathrm{C}$ stretching vibration of the ether linkage (Song et al. 2017) underwent notable shifts in the presence of CNCs. Methyl and hydroxypropyl groups adjacent to the ether linkage play crucial role in supramolecular organisation of HPMC-based systems (Joshi 2011). Involvement of CNCs in interactions with these groups is expected to have effect on $\mathrm{C}-\mathrm{O}-\mathrm{C}$ vibrations. The incorporation of 
the hydrophilic CNCs led to a blue shift of the band (from $947.1 \mathrm{~cm}^{-1}$ to $949.5 \mathrm{~cm}^{-1}$, Figure $\mathrm{S} 6 \mathrm{~b}$ of Supplementary Material) while octyl-CNCs caused a red shift to $944.8 \mathrm{~cm}^{-1}$. Thus higher energy is required to induce excited $\mathrm{C}-\mathrm{O}-\mathrm{C}$ stretching of HPMC when it interacts with octyl-CNCs. However, excitation in HPMC/CNCs occurred when the energy absorbed was even smaller than for HPMC alone. This might be an indication that hydrophilic CNCs interfere with intermolecular hydrogen bonding between HPMC macromolecules; this facilitates $\mathrm{C}-\mathrm{O}-\mathrm{C}$ stretching vibrations. On the other hand, the association of octyl-CNCs with methyl and hydroxypropyl groups via hydrophobic interactions impedes the stretching vibrations since a bulky nanoparticle became physically bound to the alkyl groups adjacent to the ether linkage.

The transient nature of physical cross-links in hydrogel networks is a prerequisite trait for selfhealing materials. Both HPMC and hybrid HPMC/ CNCs hydrogels showed the ability to restore their rheological properties after strain-induced rupture (rotational shear) of their structure (Figure S7 of Supplementary Material). For the weak network of HPMC, structural recovery occurred within $2 \mathrm{~min}$ after stress removal. However, longer times were required to restore rheological properties for more complex hybrid networks with slower recovery observed for the HPMC/octyl-CNC hydrogel compared with HPMC/CNC. Although $90 \%$ of the $\mathrm{G}^{\prime}$ recovered in approximately $3 \mathrm{~min}$, almost $15 \mathrm{~min}$ was needed for full recovery values for the HPMC/octylCNC hydrogel. This is probably due to a slower assembling process involving nanoparticles and soluble polymer in the systems of high viscosity.

Rheological properties of the hybrid supramolecular colloidal hydrogel are expected to depend on the polymer/nanoparticle ratio and the total solids concentration, which would allow formulating hydrogels with different rheological properties. In Fig. 2b, frequency dependencies of $\mathrm{G}^{\prime}$ as an indicator of hydrogel stiffness and strength are presented for several compositions. All hydrogels had the same solids content of $4 \mathrm{wt} \%$. The increase of octyl-CNC/ HPMC ratio resulted in the enhancement of the hydrogel stiffness accompanied with a lesser dependence on frequency (decrease in power low index $(n)$ $\left.\mathrm{G}^{\prime} \sim \omega^{n}\right)$. Further evidence for the formation of a stronger network is obtained in large amplitude oscillatory shear (LAOS) experiments. Four types of $\mathrm{G}^{\prime}$ and $\mathrm{G}^{\prime \prime}$ response in LAOS experiments have been identified (Hyun et al. 2002): type I, strain thinning when both moduli decrease with the increase of strain; type II, strain hardening (increase of both modulus at larger strains); type III, weak strain overshoot $\left(\mathrm{G}^{\prime}\right.$ decreases with the increase of strain while $G^{\prime \prime}$ increases in a certain range of shear followed by a decrease); type IV, strong strain overshoot (both moduli exhibit an increase in a certain strain range followed by a decrease). Figure S8a in Supplementary Material demonstrates that at $20{ }^{\circ} \mathrm{C}$ strain thinning (type I) was a characteristic of all hydrogels, except the HPMC/Octyl-CNC 25/75 hydrogel. The latter showed a segment of rising $\mathrm{G}^{\prime \prime}$ followed by a decrease at larger strains. Such a response falls into type III; namely a weak strain overshoot. $G^{\prime \prime}$ indicates the energy dissipation due to a reorganization of the material's structure. The increase in $\mathrm{G}^{\prime \prime}$ for the HPMC/ Octyl-CNC 25/75 hybrid hydrogel implies that an additional energy is required for the distortion of the network structure which is in opposition to the flow. Thus, the hybrid network is the strongest among the studied compositions. When the network of HPMC/ Octyl-CNC 25/75 hydrogel became fragmented by large deformations, a decrease in $\mathrm{G}^{\prime \prime}$ is observed due to the alignment of polymer chains and nanoparticles.

In addition to oscillatory measurements, the hydrogels were characterized in steady shear viscometry (Fig. 3, Figure S9 Supplementary Material). As shown in Fig. 3, all hydrogels demonstrated shear-thinning behavior reflecting the transient state of physical crosslinks. Generally, the Carreau model was a good fit to the experimental data acquired at $20{ }^{\circ} \mathrm{C}$. This model is defined by the equation

$\frac{\eta_{\dot{\gamma}}-\eta_{\infty}}{\eta_{0}-\eta_{\infty}}=\frac{1}{1+(K \dot{\gamma})^{1-n}}$

where $\eta_{0}$ and $\eta_{\infty}$ are the zero-shear rate viscosity and an infinite shear rate viscosity, respectively, and $K$ and $n$ are fitting parameters. Figure 3 presents the fitted curves and corresponding values of the zero-shear rate viscosity for the hydrogels of several compositions. In line with the results of oscillatory measurements the zero-shear viscosity increased with an increasing content of octyl-CNCs in the hybrid hydrogels. The increase, of two order of magnitude was observed for 


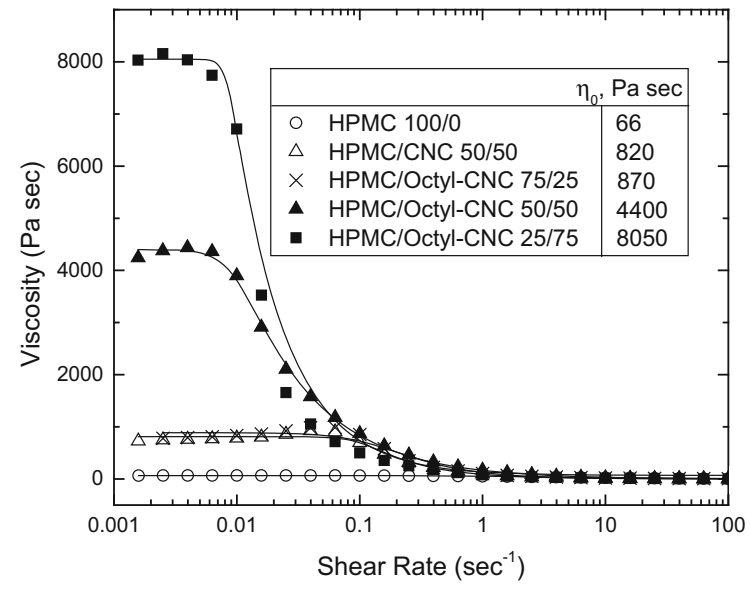

Fig. 3 Typical flow curves for HPMC hydrogel and hybrid hydrogels with CNCs or octyl-CNCs of different compositions. The total solids concentration was $4 \mathrm{wt} \%$. The numbers given in the compositions represent the mass ratio between the HPMC and the CNCs. Solid lines are the Carreau model $\left(\mathrm{R}^{2}>0.9\right.$ for all compositions). Zero-shear viscosity is obtained from the fitted curves

hybrid HPMC/Octyl-CNCs 25/75 hydrogels compared with the pure HPMC hydrogels. A growing number of active junctions between HPMC molecules and octyl-CNCs with an increase of the nanoparticle content is thought to form more mechanically robust networks. This leads to higher values of shear viscosity. However, physical crosslinks are disturbed by the flow leading to their dissociation and consequently a drop in the viscosity with an increase in the shear rate.

Thermal response of hybrid hydrogels

It is well known that hydrophobic interactions are entropically driven, although that depends on the size of the solute. They are highly sensitive to temperature change, and as such should contribute to the variations in hydrogel properties with temperature. The thermal response of HPMC is well documented (Haque et al. 1993; Joshi 2011; Silva et al. 2008). In a temperature range between 50 and $70{ }^{\circ} \mathrm{C}$ two stages in the transformation of HPMC solutions have been identified: (1) clustering of hydrophobic domains which is accompanied with the decrease in $\mathrm{G}^{\prime}$; (2) phase separation with the formation of interconnected polymer-rich regions and polymer-depleted regions. The system transforms into a gel state during the second stage, which manifests itself by sharp increase in $\mathrm{G}^{\prime}$ during oscillatory rheometry. The change of $\mathrm{G}^{\prime}$ with temperature obtained for the $4 \mathrm{wt} \%$ HPMC solution (Fig. 4) complies with these observations; $\mathrm{G}^{\prime}$ gradually decreased with an increase in temperature from 20 to $60{ }^{\circ} \mathrm{C}$. Above $60{ }^{\circ} \mathrm{C}$, an abrupt decrease in $\mathrm{G}^{\prime}$ was observed followed by sharp $\mathrm{G}^{\prime}$ increase when the temperature exceeded $65{ }^{\circ} \mathrm{C}$. However, only a minor decrease in $\mathrm{G}^{\prime}$ was observed for hybrid hydrogels with hydrophilic $\mathrm{CNCs}$ in the temperature range between 20 and $50{ }^{\circ} \mathrm{C}$. At the same time, $\mathrm{G}^{\prime}$ was found to increase even at low temperatures when the hydrogels contained octyl-CNCs. These observations further confirm the participation of nanoparticles in the formation of a hybrid network structure.

The association of octyl-CNCs is likely to occur even at low temperatures, as was demonstrated for the thermal response of surface tension. This effect combined with hydrophobic interactions between octyl-CNCs and HPMC resulted in a continuous increase in $\mathrm{G}^{\prime}$ for the hybrid hydrogels containing octyl-CNCs. The most significant changes in the rheological properties occurred at temperatures greater than $55^{\circ} \mathrm{C}$. It appears that the onset of these changes is not affected by the type of CNCs used; hydrophilic or hydrophobized CNCs. Over the whole temperature range, the association of HPMC and

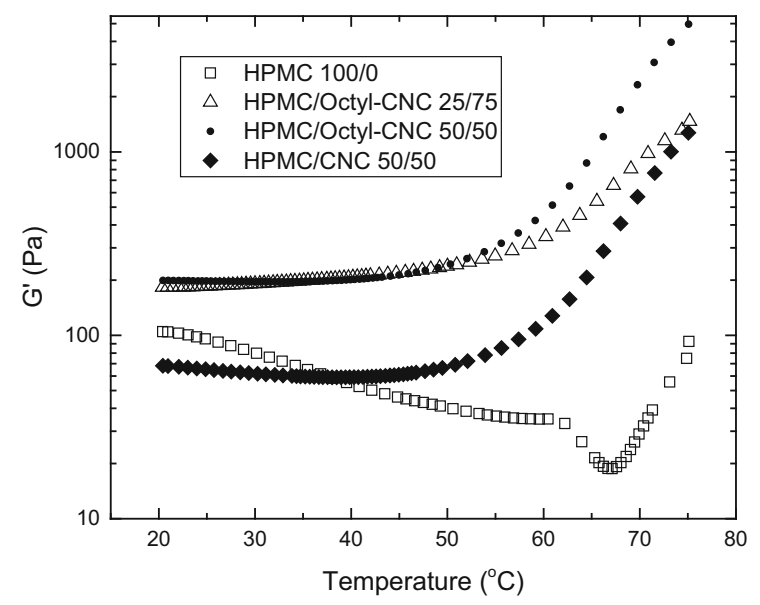

Fig. 4 Variation of storage modulus as a function of temperature for HPMC and hybrid hydrogels containing $\mathrm{CNCs}$ or octyl-CNCs. Total solids concentration was $4 \mathrm{wt} \%$. The numbers shown in the compositions represent the mass ratio between HPMC and the CNCs. Angular frequency was $6.28 \mathrm{rad}$ $\mathrm{s}^{-1}$ and the strain amplitude was set at $1.5 \%$ 
octyl-CNCs via hydrophobic interactions yielded hybrid hydrogels of higher stiffness compared to materials containing hydrophilic CNCs.

LAOS experiments at $50{ }^{\circ} \mathrm{C}$, presented in Figure $\mathrm{S} 8 \mathrm{~b}$ of Supplementary Material, revealed a type III response for HPMC/octyl-CNC hydrogels of 25/75 and 50/50 compositions while HPMC solutions and hybrid hydrogels with hydrophilic CNCs or low content octyl-CNC (HPMC/octyl-CNCs 75/25) remained strain thinning at elevated temperature. Thus, temperature induced association of HPMC in the solutions on its own, or in combination with hydrophilic CNC does not form strong enough structures to be detectable with an increase in amplitude strain. In contrast to low temperature (Figure S8a, Supplementary Material) HPMC/octyl-CNCs 50/50 showed an increase in $\mathrm{G}^{\prime \prime}$. Notably higher increase in $\mathrm{G}^{\prime \prime}$ for the HPMC/octyl-CNCs 25/75 composition and transformation of HPMC/octyl-CNCs 50/50 into type III signify the effect of temperature on the strength of the hybrid network. The higher network strength causes more energy dissipation in LAOS at a shear which induces network disruption. Interestingly, for hydrogels with a higher octyl-CNC content (HPMC/ Octyl-CNC 25/75) smaller increases in stiffness were observed at higher temperatures (Fig. 4). It is probable that when the hydrogels contain a large fraction of octyl-CNCs the temperature induced self-association becomes dominant and a smaller number of physical crosslinks with HPMC are formed. This results in a less pronounced stiffening of the hydrogels with an increase of temperature. Thus, to obtain optimal properties of the hydrogels, the content of the soluble polymer and CNCs must be balanced depending on the temperature and hydrogel properties required for a specific application.

Flow curves also were obtained for the hydrogels at temperatures in the range $20-60{ }^{\circ} \mathrm{C}$ and are presented in Supplementary Material (Figure S9). Hybrid hydrogels exhibited a narrow shear thickening region at low shear rates, especially at elevated temperatures. (Note: the increase in viscosity is not obvious on all the curves due to the use of a $\log$ scale). Such a response to shear has been previously observed for some polyelectrolytes and the mechanism of this type of shearthickening is not clear (Benchabane and Bekkour 2008). These data cannot be fitted using the Carreau model. Therefore, in order to compare the temperature

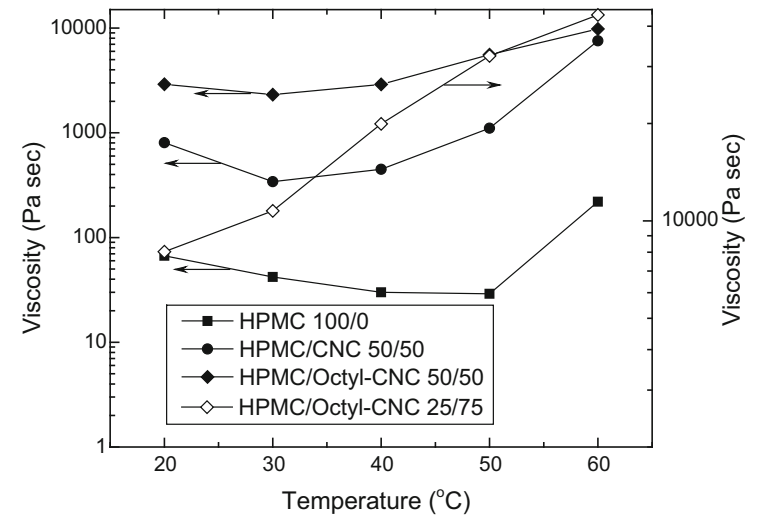

Fig. 5 The influence of temperature on hydrogel viscosity at a shear rate of $0.015 \mathrm{~s}^{-1}$. Total solids concentration was $4 \mathrm{wt} \%$. The numbers in the compositions represent the mass ratio between HPMC and CNCs

effect on the viscosity of hydrogels, Fig. 5 presents results for viscosities taken from the flow curves in the region of small shear; namely $0.015 \mathrm{~s}^{-1}$. Similar to changes in $\mathrm{G}^{\prime}$, the viscosity at this shear rate responded to temperature in a complex manner. A clear decrease in viscosity was observed for the HPMC hydrogel and the hybrid hydrogel containing CNCs for temperatures up to $40{ }^{\circ} \mathrm{C}$. This is thought to be a result of a commonly seen decrease in a fluids' viscosity with an increase in temperature. Relatively small reinforcement with hydrophilic CNCs cannot compete with this effect within the low temperature range; viscosity starts increasing only when the association of HPMC becomes significant at temperatures greater than $50{ }^{\circ} \mathrm{C}$. However, when hydrophobic octyl-CNCs are used, there was only a very small initial decrease in viscosity for HPMC/octyl-CNCs 50/50, while a steady increase in viscosity was observed for HPMC/octylCNCs 25/75.

Further insights into hydrogel structuring and thermal response were obtained from saturation transfer difference (STD) NMR spectroscopy experiments by assessing binding of water to the hydrogel network (Calabrese et al. 2019). The analysis of the STD initial slope $\left(\mathrm{STD}_{0}\right)$ of the residual water peak HDO (see Experimental section) reports on the fraction of bound HDO (Fig. 6). The gradual increase of the HDO $\mathrm{STD}_{0}$ from 30 to $55^{\circ} \mathrm{C}$ for mixtures of HPMC with both types of CNCs suggests an increased fraction of water bound by the network upon heating (Fig. 6), which qualitatively correlates with the 


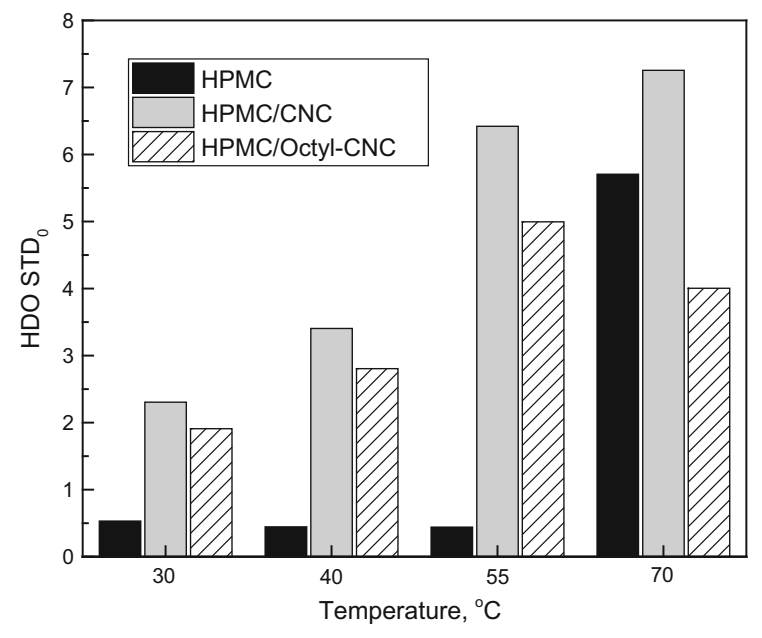

Fig. 6 Bar chart showing the evolution of the $\mathrm{STD}_{0}$ of the residual water peak (HDO) in HPMC solution, HPMC/CNC 50/50 and HPMC/Octyl-CNC 50/50 with total solid content 1 $\mathrm{wt} \%$ as a function of temperature, irradiating at $0 \mathrm{ppm}$ (corresponding to the NMR invisible "core" of cellulose)

increase in storage modulus $\left(\mathrm{G}^{\prime}\right)$ and viscosity (Figs. 4, 5, respectively). For the HPMC solution, however, the value of $\mathrm{STD}_{0}$ of HDO decreases slightly from 30 to $55{ }^{\circ} \mathrm{C}$, which might be associated with either a partial desolvation of macromolecules and/or less efficient spin diffusion at higher temperatures. It should be noted that the significant increase of the HDO STD 0 observed from 55 to $70{ }^{\circ} \mathrm{C}$ is due to the HPMC sample undergoing a sol-to-gel transition at around $65^{\circ} \mathrm{C}$ (crossing of $\mathrm{G}^{\prime}$ and $\mathrm{G}^{\prime \prime}$, Figure $\mathrm{S} 10$ of Supplementary Material). Therefore, the increased fraction of network-bound water in the HPMC/CNCs and HPMC/Octyl-CNCs mixtures might be an important parameter to consider when explaining the growth of $\mathrm{G}^{\prime}$ and viscosity upon heating. In that sense, it is relevant to note that the temperature dependence of $\mathrm{HDO} \mathrm{STD}_{0}$ is higher for HPMC/CNC than HPMC/ Octyl-CNC hybrid hydrogels, or in other words, the fraction of network-bound water showed a higher increase for HPMC/CNC upon heating up to $70{ }^{\circ} \mathrm{C}$ (Fig. 6). Moreover, HDO $\mathrm{STD}_{0}$ was consistently lower for hydrogels incorporating hydrophobized octyl-CNCs compared with the HPMC/CNC hydrogel. This decrease to a certain degree is likely to be defined by a smaller area of hydrophilic surface for octyl-CNCs. However, association of hydrophobic domains in HPMC/octyl-CNC and subsequent water exclusion might also contribute to the decrease of bound water in HPMC/octyl-CNC systems. It is possible that $\mathrm{HDO} \mathrm{STD}_{0}$ decrease observed with the increase of temperature from 55 to $70{ }^{\circ} \mathrm{C}$ augmented this contribution due to extensive association of hydrophobic domains. Thus, the differences in the behavior of the HDO $\mathrm{STD}_{0}$ between both hybrid hydrogels upon heating might result from a reduced water accessible surface area in the HPMC/Octyl$\mathrm{CNC}$ network due to increased aggregation promoted by octyl-CNC hydrophobic interactions. This agrees with the much higher elastic behavior $\left(\mathrm{G}^{\prime}\right)$ of HPMC/ Octyl-CNC within the whole temperature range (Fig. 4).

Formation of hybrid supramolecular and colloidal hydrogels of HPMC with CNCs and their thermal response are illustrated in Fig. 7 which is based on a model proposed by $\mathrm{Li}$ et al. (2001) for sol-to-gel transition for methylcellulose. At low temperatures HPMC macromolecules can have weak hydrophobic association points. However, the number of these points cannot result in the formation of a continuous network. Although hydrophilic CNCs can interact with HPMC chains via hydrogen bonding and van der Waals interactions, strong repulsion between surfacecharged CNCs leads to a uniform distribution of CNC nanoparticles without their aggregation (Fig. 7a). CNCs do not contribute to the formation of transient physical cross-links. In contrast, due to an ability of octyl-CNCs to self-associate, and the association with hydrophobic domains of HPMC driven by hydrophobic interactions, a continuous hybrid network forms in HPMC/octyl-CNC systems at low temperatures (Fig. 7b). As a result, HPMC/Octyl-CNC gels are significantly stiffer, and more viscous than HPMC/ CNCs gels. Exclusion of water from hydrophobic junctions causes a decrease in the amount of bound water in HPMC/Octyl-CNC (Fig. 6). This is expected to result in a higher density of the polymer rich phase in HPMC/Octyl-CNC hydrogels. SEM images (Fig. 7 bottom panel) reveals a smaller cell size in the HPMC/ CNC hydrogel compared with HPMC/octyl-CNC. This might be an indication of such densification of a polymer rich phase since HPMC content is the same in both hydrogels and thinner portioning walls should form structures with smaller cell sizes. However, one must use cryo-SEM results with caution for the interpretation of hydrogel structure due to possible 

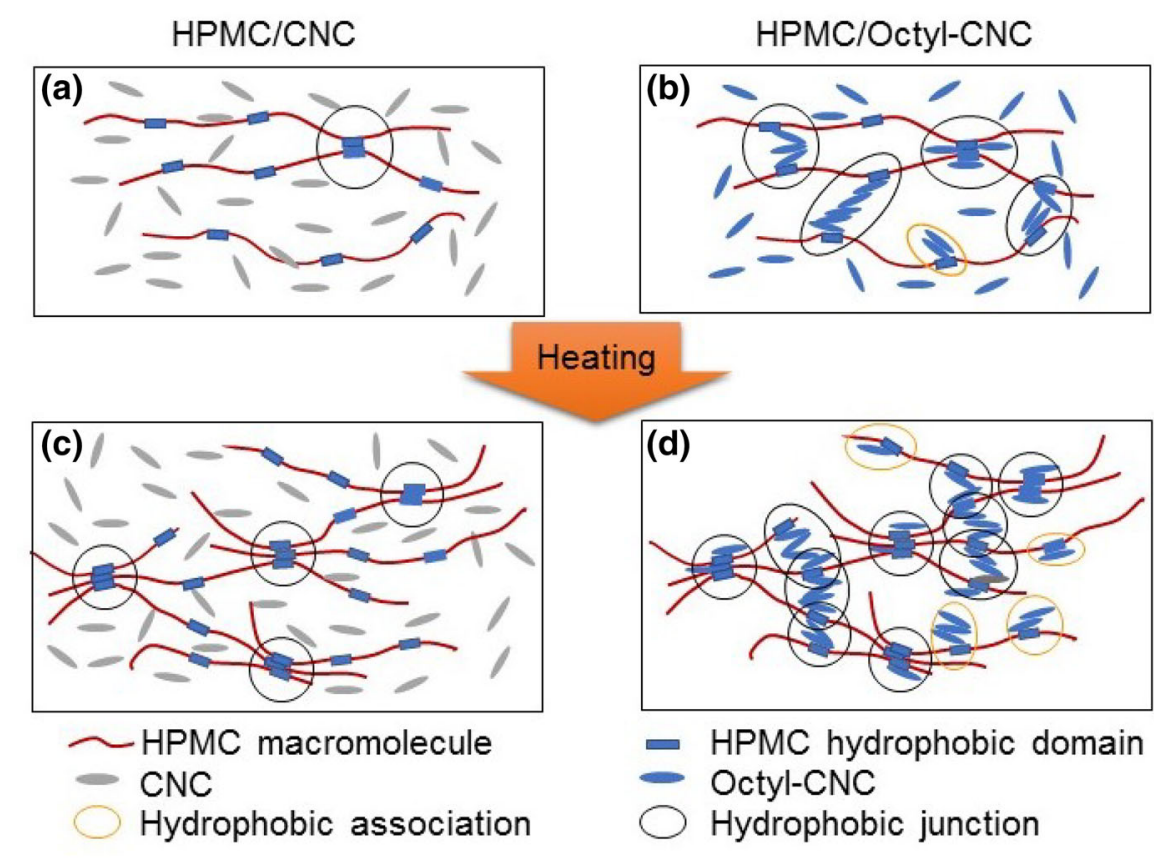

Heating
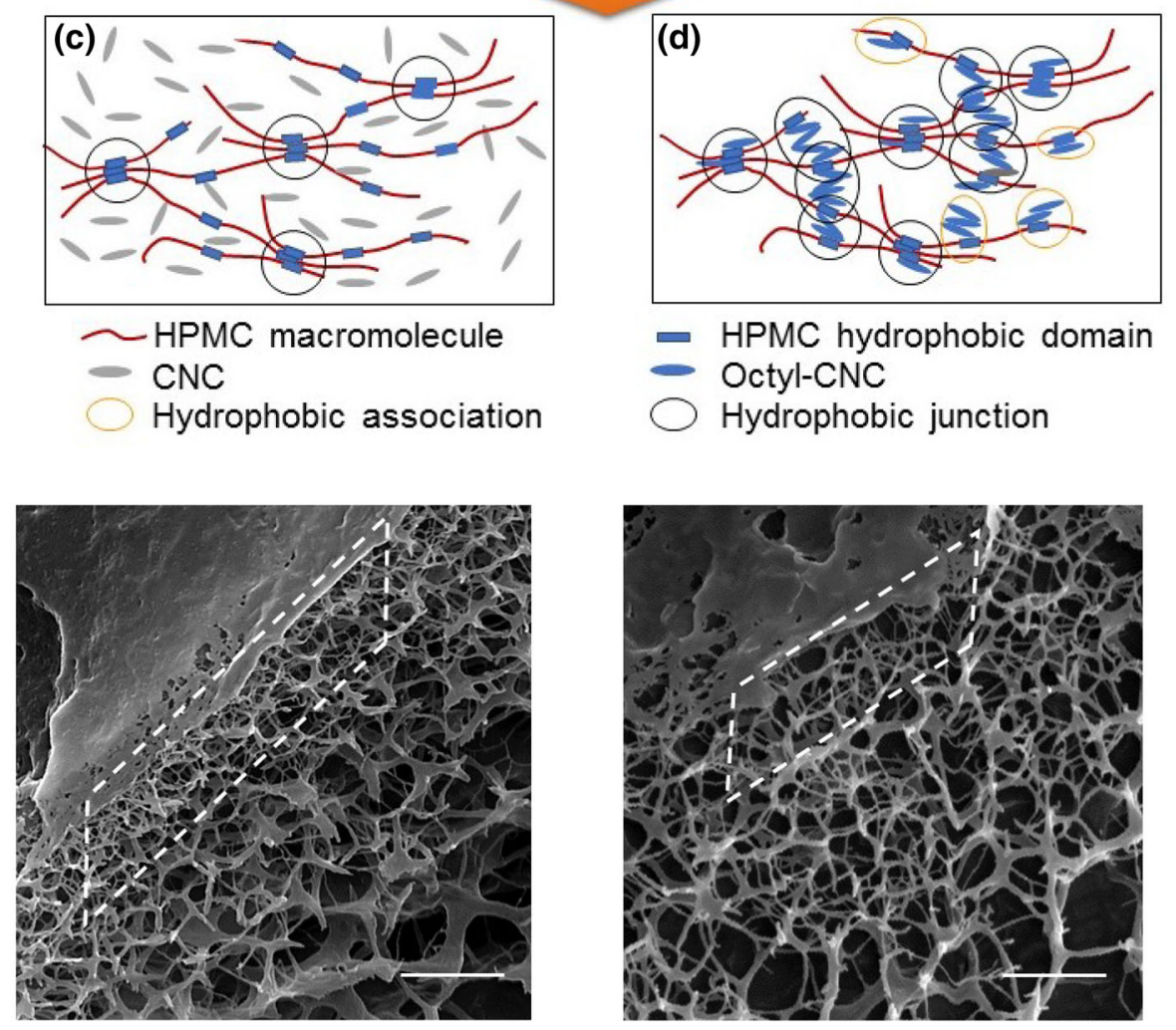

Fig. 7 Structure of hybrid hydrogels. Top panel: Schematic drawing of composite gel formation driven by hydrophobic interactions and evolution of hydrogel networks with heating.

artefacts of hydrogel dehydration (Draper and Adams 2018).

Upon heating, hydrophobic association intensifies in both systems, however, in HPMC/CNC gels only hydrophobic domains of soluble HPMC participate in the formation of the hydrophobic junctions. Hydrophobic octyl-CNCs induce the formation significantly larger number of transient cross-links in HPMC/Octyl-CNC gels compared with HPMC/CNC gels at elevated temperatures as shown in Fig. $7 \mathrm{c}$, d.

Bottom panel: SEM images of corresponding cryogels (scale bar $1 \mu \mathrm{m})$. Note: the selected areas underwent the fastest cooling, therefore, better represent the structure of the hydrogels

This leads to stiffer, more viscous gels incorporating both types of CNCs at higher temperatures. However, higher density of transient cross-links maintains larger stiffness and viscosity of HPMC/octyl-CNC gels compared with HPMC/CNC gels in the whole temperature region. According to the proposed scheme a mesh size of the network decreases with the increase of temperature. This forms in a more confined space where a larger fraction of water is associated with the 
hybrid network. Thus, the increase in bound water upon heating is attributed to these structural changes.

\section{Conclusions}

CNCs-high aspect ratio nanoparticles-were hydrophobized by surface binding of octylamine. Octyl-CNCs exhibited a typical amphiphilic behavior, decreasing surface tension in the aqueous suspensions. An increase in temperature of octyl-CNC suspensions induces increases in surface tension which indicates the intensification of self-assembly of octyl-CNCs in the bulk of the suspension. Such properties of hydrophobized CNCs are a prerequisite for recently conceptualized self-assembly of hybrid hydrogels via hydrophobic interactions between amphiphilic polymers and particles.

Both hydrophilic CNCs and octyl-CNCs reinforce HPMC solutions transforming them in self-supporting hydrogels with the elastic modulus dominating the loss modulus in oscillatory rheology. This implies that both types of CNCs were incorporated into the hybrid networks. However, hydrogels with octyl-CNCs are stronger, stiffer and more viscous compared with hydrogels containing hydrophilic CNCs. Thus, assembly of the water-soluble polymer and hydrophobized nanoparticles driven by hydrophobic interactions results in the formation of a more robust hybrid network. The strength of the network formed via association of hydrophobic domains is further augmented at elevated temperatures as evidenced by a significant enhancement of rheological properties in both oscillatory rheology and steady shear viscometry. Rheological properties depend on the polymer/CNC ratio and temperature; compositions with the superior rheological properties observed at lower temperature might show inferior properties at higher temperatures. This was observed for the compositions HPMC/octylCNC 25/75 and HPMC/octyl-CNC 50/50. Thus, the polymer/octyl-CNC ratio must be balanced depending on the temperature and rheological properties required for a specific application. NMR spectroscopy revealed higher fraction of bound water in hybrid hydrogels compared with HPMC. The amount of network-bound water increases with temperature in parallel with hydrogel stiffness, strength and viscosity. This work expands the foundation for design of self-healing stimuli-responsive hybrid hydrogels with different rheological properties using assembly of amphiphilic polymers and nanoparticles driven by hydrophobic interactions.

Acknowledgments The Engineering and Physical Sciences Research Council (EPSRC) is acknowledged for provision of financial support (EP/N03340X/2, EP/N033337/1). We are grateful for the use of the University of East Anglia (UEA) Faculty of Science NMR facility and the Wolfson Bioimaging Facility of the University of Bristol. We would like to thank Mrs Judith Mantell for the cryo-SEM micrographs of the hydrogels.

Open Access This article is distributed under the terms of the Creative Commons Attribution 4.0 International License (http:// creativecommons.org/licenses/by/4.0/), which permits unrestricted use, distribution, and reproduction in any medium, provided you give appropriate credit to the original author(s) and the source, provide a link to the Creative Commons license, and indicate if changes were made.

\section{References}

Alam A, Zhang Y, Kuan H-C, Lee S-H, Maa J (2018) Polymer composite hydrogels containing carbon nanomaterials: morphology and mechanical and functional performance. Prog Polym Sci 77:1-18. https://doi.org/10.1016/j. progpolymsci.2017.09.001

Appel EA, del Barrio J, Loh XJ, Scherman OA (2012) Supramolecular polymeric hydrogels. Chem Soc Rev 41:6195-6214. https://doi.org/10.1039/c2cs35264h

Appel EA, Tibbitt MW, Webber MJ, Mattix BA, Veiseh O, Langer R (2015) Self-assembled hydrogels utilizing polymer-nanoparticle interactions. Nat Commun 6:6295. https://doi.org/10.1038/ncomms7295

Baumann MD, Kang CE, Tator CH, Shoichet MS (2010) Biomaterials intrathecal delivery of a polymeric nanocomposite hydrogel after spinal cord injury. Biomaterials 31:7631-7639. https://doi.org/10.1016/j.biomaterials. 2010.07.004

Benchabane A, Bekkour K (2008) Rheological properties of carboxymethyl cellulose (CMC) solutions. Colloid Polym Sci 286:1173-1180. https://doi.org/10.1007/s00396-0081882-2

Calabrese V, Muñoz-García JC, Schmitt J, Alves da Silva M, Scott JL, Angulo J, Khimyak YZ, Edler KJ (2019) Understanding heat driven gelation of anionic cellulose nanofibrils: combining STD NMR, SAXS and rheology. J Colloid Interface Sci 535:205-213. https://doi.org/10. 1016/j.compositesa.2012.02.006

Cao P-F, Mangadlao JD, Advincula RC (2015) Stimuli-responsive polymers and their potential applications in oilgas industry. Polym Rev 55:706-733. https://doi.org/10. 1080/15583724.2015.1040553

Chakraborty T, Chakraborty I, Ghosh S (2011) The methods of determination of critical micellar concentrations of the amphiphilic systems in aqueous medium. Arab J Chem 4:265-270. https://doi.org/10.1016/j.arabjc.2010.06.045 
Chassenieux C, Nicolai T, Benyahia L (2011) Rheology of associative polymer solutions. Curr Opin Colloid Interface Sci 16:18-26. https://doi.org/10.1016/j.cocis.2010.07.007

Draper ER, Adams DJ (2018) How should multicomponent supramolecular gels be characterised? Chem Soc Rev 47:3395-3405. https://doi.org/10.1039/c7cs00804j

Du X, Zhou J, Shi J, Xu B (2015) Supramolecular hydrogelators and hydrogels: from soft matter to molecular biomaterials. Chem Rev 115:13165-13307. https://doi.org/10.1021/acs. chemrev.5b00299

Haque A, Richardson RK, Morris ER, Gidley MJ, Caswell DC (1993) Thermogelation of methylcellulose. Part II: effect of hydroxypropyl substituents. Carbohydr Polym 22:175-186. https://doi.org/10.1016/0144-8617(93)90138-T

Haraguchi K (2007) Nanocomposite hydrogels. Curr Opin Solid State Mater Sci 11:47-54. https://doi.org/10.1016/j. cossms.2008.05.001

Hynninen V, Hietala S, McKee JR, Murtomäki L, Rojas OJ, Ikkala O, Nonappa NV (2018) Inverse thermoreversible mechanical stiffening and birefringence in a methylcellulose/cellulose nanocrystal hydrogel. Biomacromolecules 19:2795-2804. https://doi.org/10.1021/acs.biomac. $8 \mathrm{~b} 00392$

Hyun K, Kim SH, Ahn KH, Lee SJ (2002) Large amplitude oscillatory shear as a way to classify the complex fluids. J Non Newton Fluid Mech 55:51-65. https://doi.org/10. 1016/S0377-0257(02)00141-6

Joshi SC (2011) Sol-gel behavior of hydroxypropyl methylcellulose (HPMC) in ionic media including drug release. Materials 4:1861-1905. https://doi.org/10.3390/ ma4101861

Kalashnikova I, Bizot H, Cathala B, Capron I (2012) Modulation of cellulose nanocrystals amphiphilic properties to stabilize oil/water interface. Biomacromolecules 13:267-275. https://doi.org/10.1021/bm201599j

Li L, Thangamathesvaran PM, Yue CY, Tam KC, Hu X, Lam YC (2001) Gel network structure of methylcellulose in water. Langmuir 17:8062-8068. https://doi.org/10.1021/ la010917r

Lundberg DJ, Glass JE, Eley RR (1991) Viscoelastic behavior among HEUR thickeners. J Rheol 35:1255-1274. https:// doi.org/10.1122/1.550174

Mayer M, Meyer B (1999) Characterization of ligand binding by saturation transfer difference NMR spectroscopy. Angew Chem Int Ed 38:1784-1788. https://doi.org/10.1002/ (SICI)1521-3773(19990614)38:12\%3c1784:AIDANIE1784\%3e3.0.CO;2-Q

Mazeau K (2011) On the external morphology of native cellulose microfibrils. Carbohydr Polym 84:524-532. https:// doi.org/10.1016/j.carbpol.2010.12.016

McKee JR, Hietala S, Seitsonen J, Laine J, Kontturi E, Ikkala O (2014) Thermoresponsive nanocellulose hydrogels with tunable mechanical properties. ACS Macro Lett 3:266-270. https://doi.org/10.1021/mz400596g

Mihajlovic M, Staropoli M, Appavou M-S, Wyss HM, PyckhoutHintzen W, Sijbesma RP (2017) Tough supramolecular hydrogel based on strong hydrophobic interactions in a multiblock segmented copolymer. Macromolecules 50:3333-3346. https://doi.org/10.1021/acs.macromol.7b00319

Nigmatullin R, Harniman R, Gabrielli V, Muñoz-García JC, Khimyak YZ, Angulo J, Eichhorn SJ (2018) Mechanically robust gels formed from hydrophobized cellulose nanocrystals. ACS Appl Mater Interfaces 10:19318-19322. https://doi.org/10.1021/acsami.8b05067

Sahoo JK, Van den Berg MA, Webber MJ (2018) Injectable network biomaterials via molecular or colloidal self-assembly. Adv Drug Deliv Rev 127:185-207. https:// doi.org/10.1016/j.addr.2017.11.005

Schexnailder P, Schmidt G (2009) Nanocomposite polymer hydrogels. Colloid Polym Sci 287:1-11. https://doi.org/10. 1007/s00396-008-1949-0

Silva SMC, Pinto FV, Antunes FE, Miguel MG, Sousa JJS, Pais AACC (2008) Aggregation and gelation in hydroxypropylmethyl cellulose aqueous solutions. J Colloid Interface Sci 327:333-340. https://doi.org/10.1016/j.jcis. 2008.08.056

Song B, Lu D, Peng M, Li X, Zou Y, Huang M, Lu F (2017) Fast discrimination of hydroxypropyl methyl cellulose using portable Raman spectrometer and multivariate methods. Laser Phys 27:025701. https://doi.org/10.1088/1555-6611/ aa4ffc

Tuncaboylu DC, Sari M, Oppermann W, Okay O (2011) Tough and self-healing hydrogels formed via hydrophobic interactions. Macromolecules 44:4997-5005. https://doi.org/ $10.1021 / \mathrm{ma} 200579 \mathrm{v}$

Voorhaar L, Hoogenboom R (2016) Supramolecular polymer networks: hydrogels and bulk materials. Chem Soc Rev 45:4013-4031. https://doi.org/10.1039/c6cs00130k

Zakharova L, Mirgorodskaya A, Gaynanova G, Kashapov R, Pashirova T, Vasilieva E, Zuev Yu, Synyashin O (2016) Supramolecular strategy of the encapsulation of lowmolecular-weight food ingredients. In: Grumezescu AM (ed) Encapsulations. Volume 2 nanotechnology in the agrifood industry. Academic Press, Cambridge, pp 295-362

Zheng YJ, Loh XJ (2016) Natural rheological modifiers for personal care. Polym Adv Technol 27:1664-1679. https:// doi.org/10.1002/pat.3822

Publisher's Note Springer Nature remains neutral with regard to jurisdictional claims in published maps and institutional affiliations. 